\title{
Generalized intuitionistic fuzzy ideals of hemirings
}

\author{
Asim Hussain and Muhammad Shabir \\ Quaid-i-Azam University, Islamabad, Pakistan \\ Received: 31 July 2015, Revised: 1 August 2015, Accepted: 27 August 2015 \\ Published online: 10 April 2016
}

\begin{abstract}
In this paper we generalize the concept of quasi-coincident of an intuitionistic fuzzy point with an intuitionistic fuzzy set and define $\left(\in, \in \vee q_{k}\right)^{*}$-intuitionistic fuzzy ideals of hemirings and characterize different classes of hemirings by the properties of these ideals.
\end{abstract}

Keywords: Intuitionistic fuzzy sub-hemiring, intuitionistic fuzzy ideal, fully idemotent hemiring, regular hemiring.

\section{1 introduction}

Dedekind introduced the modern definition of the ideal of a ring in 1894 and observed that the family $\operatorname{Id}(R)$ of all the ideals of a ring $R$ obeyed most of the rules that the $\operatorname{ring}(R,+, \cdot)$ did, but $(\operatorname{Id}(R),+, \cdot)$ was not a ring. In 1934, Vandiver [25] studied an algebraic system, which consists of a non-empty set $S$ with two binary operations "+" and "." such that $S$ was semigroup under both the operations and $(S,+, \cdot)$ satisfies both the distributive laws but did no satisfy the cancellation law of addition. Vandiver named this system a 'semiring'. Semirings are common generalization of rings and distributive lattices. A hemiring is a semiring in which " + " is commutative and it has an absorbing element. Semirings (hemirings) appear in a natural manner in some applications to the theory of automata, formal languages, optimization theory and other branches of applied mathematics (see for example [9,10,11,12,18, 19] ).

Zadeh introduced the concept of fuzzy set in his definitive paper [26] of 1965. Many authors used this concept to generalize basic notions of algebra. In 1971, Rosen feld [22] laid the foundations of fuzzy algebra. He introduced the notions of fuzzy subgroup of a group. Ahsan et al. [3] initiated the study of fuzzy semirings. Murali [20] defined the concept of belongingness of a fuzzy point to a fuzzy subset under a natural equivalence on fuzzy subset and Pu and Liu introduced the concept of quasicoincident of a fuzzy point with a fuzzy set in [21]. Bhakat and Das [5] used these ideas and defined $(\in, \in \vee q)$-fuzzy subgroup of a group which is a generalization of Rosenfeld's fuzzy subgroup. Many researchers used these ideas to define $(\alpha, \beta)$-fuzzy substructures of algebraic structures (see $[8,15,16,23])$.

Generalizing the concept of the quasi-coincident of a fuzzy point with a fuzzy subset, Jun [13] defined $\left(\in, \in \vee q_{k}\right)$-fuzzy subalgebra in BCK/BCI-algebras. In [24] Shabir et al. characterized semigroups by the properties of $\left(\in, \in \vee q_{k}\right)$-fuzzy ideals, quasi-ideal and bi-ideals. Jun et al. in [15] defined $\left(\in, \in \vee q_{k}\right)$-fuzzy ideals of hemirings. Asghar et al. [17], defined $\left(\in, \in \vee q_{k}\right)$-fuzzy bi-ideals in ordered semigroups.

On the other hand Atanassov [4] introduced the notion of intuitionistic fuzzy set which is a generalization of fuzzy set. Intuitionistic fuzzy hemirings are studied by Dudek in [7]. Coker and Demirici [6] introduced the notion of fuzzy point. In [14], Jun introduced the notion of $(\phi, \psi)$-intuitionistic fuzzy subgroup of a intuitionistic group where 
$\phi, \psi \in\{\in, q, \in \vee q, \in \wedge q\}$ and $\phi \neq \in \wedge q$.

Generalizing the concept of quasi-coincident of an intuitionistic fuzzy point with an intuitionistic fuzzy set we define $\left(\in, \in \vee q_{k}\right)^{*}$-intuitionistic fuzzy ideals of hemirings and characterize different classes of hemirings by the properties of these ideals.

\section{Preliminaries}

A semiring is a set $R$ together with two binary operations addition "+" and multiplication "." such that $(R,+)$ and $(R, \cdot)$ are semigroups, where both algebraic structures are connected by the ring like distributive laws:

$$
a(b+c)=a b+a c \text { and }(a+b) c=a c+b c
$$

for all $a, b$ and $c \in R$. An element $0 \in R$ is called a zero element of $R$ if $a+0=0+a=a$ and $0 \cdot a=a \cdot 0=0$ for all $a \in R$. A hemiring is a semiring with zero element, in which " + " is commutative. A hemiring $(R,+, \cdot)$ is called commutative if multiplication is commutative, that is $a b=b a$ for all $a, b \in R$. An element $1 \in R$ is called an identity element of $R$ if $a \cdot 1=1 \cdot a=a$ for all $a \in R$. A non-empty subset $I$ of a hemiring $R$ is called a left (right) ideal of $R$ if $I$ is closed under addition and $R I \subseteq I(I R \subseteq I)$. I is called a two-sided ideal or simply an ideal of $R$ if $I$ is both a left ideal and a right ideal of $R$. A hemiring $R$ is called regular if for each $x \in R$ there exists $a \in R$ such that $x=x a x$.

Theorem 1. [1] $A$ hemiring $R$ is regular if and only if $A \cap B=A B$ for all right ideals $A$ and left ideals $B$ of $R$. Generalizing the concept of regular hemirings, in [2] right weakly regular hemirings are defined as: A hemiring $R$ is right weakly regular if for each $x \in R$, we have $x \in(x R)^{2}$. If $R$ is commutative then the concepts of regular and right weakly regular coincides. It is proved in [2].

Theorem 2. [2] The following conditions are equivalent for a hemiring $R$ with 1.

(1) $R$ is right weakly regular.

(2) $A \cap B=A B$ for all right ideals $A$ and two-sided ideals $B$ of $R$.

(3) $A^{2}=A$ for every right ideal $A$ of $R$.

If $R$ is commutative, then the above conditions are equivalent to

(4) $R$ is regular.

Let $X$ be a non-empty fixed set. An intuitionistic fuzzy subset $A$ of $X$ is an object having the form

$$
A=\left\{\left\langle x, \mu_{A}(x), \lambda_{A}(x): x \in X\right\rangle\right\}
$$

where the functions $\mu_{A}: X \longrightarrow[0,1]$ and $\lambda_{A}: X \longrightarrow[0,1]$ denote the degree of membership (namely $\mu_{A}(x)$ ) and the degree of nonmembership (namely $\lambda_{A}(x)$ ) of each element of $x \in X$ to $A$, respectively, and $0 \leq \mu_{A}(x)+\lambda_{A}(x) \leq 1$ for all $x \in X$. For the sake of simplicity, we use the symbol $A=\left(\mu_{A}, \lambda_{A}\right)$ for the intuitionistic fuzzy subset (briefly, IFS) $A=\left\{\left\langle x, \mu_{A}(x), \lambda_{A}(x): x \in X\right\rangle\right\}$. If $A=\left(\mu_{A}, \lambda_{A}\right)$ and $B=\left(\mu_{B}, \lambda_{B}\right)$ are intuitionistic fuzzy subsets of $X$, then

(1) $A \subseteq B \Longleftrightarrow \mu_{A}(x) \leq \mu_{B}(x)$ and $\lambda_{A}(x) \geq \lambda_{B}(x) \quad \forall x \in X$

(2) $A=B \Longleftrightarrow A \subseteq B$ and $B \subseteq A$.

(3) $\bar{A}=\left(\lambda_{A}, \mu_{A}\right)$. More generally if $\left\{A_{i}: i \in I\right\}$ is a family of intuitionistic fuzzy subset of $X$, then by the union and intersection of this family we mean an intuitionistic fuzzy subsets

(4) $\bigcup_{i \in I} A_{i}=\left(\bigvee_{i \in I} \mu_{A_{i}}, \wedge \lambda_{i \in I}\right)$. 
(5) $\bigcap_{i \in I} A_{i}=\left(\bigwedge_{i \in I} \mu_{A_{i}}, \bigvee_{i \in I} \lambda_{A_{i}}\right)$.

Let $a$ be a point in a non-empty set $X$. If $\alpha \in(0,1]$ and $\beta \in[0,1)$ are two real numbers such that $0 \leq \alpha+\beta \leq 1$ then IFS.

$$
a(\alpha, \beta)=\left\langle x, a_{\alpha}, 1-a_{1-\beta}\right\rangle
$$

is called an intuitionistic fuzzy point(IFP) in $X$, where $\alpha$ and $\beta$ is the degree of membership and nonmembership of $a(\alpha$, $\beta$ ) respectively and $a \in X$ is the support of $a(\alpha, \beta)$.

Let $a(\alpha, \beta)$ be an IFP in $X$, and $A=\left(\mu_{A}, \lambda_{A}\right)$ be an IFS in $X$. Then $a(\alpha, \beta)$ is said to belong to $A$, written $a(\alpha, \beta) \in A$, if $\mu_{A}(a) \geq \alpha$ and $\lambda_{A}(a) \leq \beta$ and quasi-coincident with $A$, written $a(\alpha, \beta) q A$, if $\mu_{A}(a)+\alpha>1$, and $\lambda_{A}+\beta<1 . a(\alpha$, $\beta) \in \vee q A$, means that $a(\alpha, \beta) \in A$ or $a(\alpha, \beta) q A$ and $a(\alpha, \beta) \in \wedge q A$, means that $a(\alpha, \beta) \in A$ and $a(\alpha, \beta) q A$ and $a(\alpha$, $\beta) \overline{\in q} A$, means that $a(\alpha, \beta) \in \vee q A$ doesn't hold.

Let $x(t, s)$ be an IFP in $X$, and $A=\left(\mu_{A}, \lambda_{A}\right)$ be an IFS in $R$, Then for all $x, y \in R$ and $t \in(0,1], s \in[0,1)$, we define the following:

(i) $x(t, s) q_{k} A$ if $\mu_{A}(x)+t+k>1$ and $\lambda_{A}(x)+s+k<1$.

(ii) $x(t, s) \in \vee q_{k} A$ if $x(t, s) \in A$ or $x(t, s) q_{k} A$.

(iii) $x(t, s) \in \wedge q_{k} A$ if $x(t, s) \in A$ and $x(t, s) q_{k} A$.

(iv) $x(t, s) \overline{\in \vee q_{k}} A$ means that $x(t, s) \in \vee q_{k} A$ doesn't hold, where $k \in[0,1)$.

\section{$3(\alpha, \beta)$-intuitionistic fuzzy ideals}

Throughout the remaining paper $k \in[0,1), \alpha$ any one of $\in, q_{k}, \in \vee q_{k}$ and $\beta$ any one of $\in, q_{k}, \in \vee q_{k}, \in \wedge q_{k}$ unless otherwise specified.

Definition 1. An IFS $A=\left(\mu_{A}, \lambda_{A}\right)$ of a hemiring $R$ is called an $(\alpha, \beta)$-intuitionistic fuzzy sub-hemiring of $R$, if $\forall x, y \in R$ and $t_{1}, t_{2} \in(0,1], s_{1}, s_{2} \in[0,1)$,

(1) $x\left(t_{1}, s_{1}\right), y\left(t_{2}, s_{2}\right) \alpha A \Rightarrow(x+y)\left(\min \left(t_{1}, t_{2}\right), \max \left(s_{1}, s_{2}\right)\right) \beta A$,

(2) $x\left(t_{1}, s_{1}\right), y\left(t_{2}, s_{2}\right) \alpha A \Rightarrow(x y)\left(\min \left(t_{1}, t_{2}\right), \max \left(s_{1}, s_{2}\right)\right) \beta A$.

Definition 2. An IFS $A=\left(\mu_{A}, \lambda_{A}\right)$ of a hemiring $R$ is called an $(\alpha, \beta)$-intuitionistic fuzzy left (right) ideal of $R$, if $\forall$ $x, y \in R$ and $t_{1}, t_{2} \in(0,1], s_{1}, s_{2} \in[0,1)$,

(1) $x\left(t_{1}, s_{1}\right), y\left(t_{2}, s_{2}\right) \alpha A \Rightarrow(x+y)\left(\min \left(t_{1}, t_{2}\right), \max \left(s_{1}, s_{2}\right)\right) \beta A$

(2) $y\left(t_{1}, s_{1}\right) \alpha A, x \in R \Rightarrow(x y)\left(t_{1}, s_{1}\right) \beta A\left((y x)\left(t_{1}, s_{1}\right) \beta A\right)$.

An IFS $A=\left(\mu_{A}, \lambda_{A}\right)$ of a hemiring $R$ is called an $(\alpha, \beta)$-intuitionistic fuzzy ideal of $R$, if it is both $(\alpha, \beta)$-intuitionistic fuzzy left ideal and $(\alpha, \beta)$-intuitionistic fuzzy right ideal of $R$.

Theorem 3. Let $A=\left(\mu_{A}, \lambda_{A}\right)$ be an $(\alpha, \beta)$-intuitionistic fuzzy ideal of $R$. Then the set

$$
R_{(0,1)}=\left\{x \in R: \mu_{A}(x)>0 \text { and } \lambda_{A}(x)<1\right\} \neq \phi
$$

is an ideal of $R$. 
Proof. Let $x, y \in R_{(0,1)}$. Then $\mu_{A}(x)>0$ and $\lambda_{A}(x)<1, \mu_{A}(y)>0$ and $\lambda_{A}(y)<1$. Assume that $\mu_{A}(x+y)=0$ or $\lambda_{A}(x+y)=1$. If $\alpha \in\left\{\in, \in \vee q_{k}\right\}$, then, $x\left(\mu_{A}(x), \lambda_{A}(x)\right) \alpha A$ and $y\left(\mu_{A}(y), \lambda_{A}(y)\right) \alpha A$ but $(x+y)\left(\min \left\{\mu_{A}(x), \mu_{A}(y)\right\}\right.$, $\left.\max \left\{\lambda_{A}(x), \lambda_{A}(y)\right\}\right) \bar{\beta} A$, for every $\beta \in\left\{\in, q_{k}, \in \vee q_{k}, \in \wedge q_{k}\right\}$, a contradiction. Also $x(1,0) q_{k} A$ and $y(1,0) q_{k} A$ but $(x+y)(1,0) \bar{\beta} A$ for every $\beta \in\left\{\in, q_{k}, \in \vee q_{k}, \in \wedge q_{k}\right\}$, a contradiction. Thus $\mu_{A}(x+y)>0$ and $\lambda_{A}(x+y)<1$. Therefore, $x+y \in R_{(0,1)}$.

Let $x \in R_{(0,1)}$ and $y \in R$. Then $\mu_{A}(x)>0$ and $\lambda_{A}(x)<1$. suppose that $\mu_{A}(x y)=0$ or $\lambda_{A}(x y)=1$. If $\alpha \in\left\{\in \in \in \vee q_{k}\right\}$, then $x\left(\mu_{A}(x), \lambda_{A}(x)\right) \alpha A$ but $(x y)\left(\mu_{A}(x), \lambda_{A}(x)\right) \bar{\beta} A$ for every $\beta \in\left\{\in, q_{k}, \in \vee q_{k}, \in \wedge q_{k}\right\}$, a contradiction. Also $x(1,0) q_{k} A$ but $(x y)(1,0) \bar{\beta} A$ for every $\beta \in\left\{\in, q_{k}, \in \vee q_{k}, \in \wedge q_{k}\right\}$, a contradiction. Thus $\mu_{A}(x y)>0$ and $\lambda_{A}(x y)<1$. Therefore, $x y \in R_{(0,1)}$. Similarly $y x \in R_{(0,1)}$. This completes the proof.

Theorem 4. Let $A=\left(\mu_{A}, \lambda_{A}\right)$ be an $(\alpha, \beta)$-intuitionistic fuzzy sub-hemiring of $R$. Then the set

$$
R_{(0,1)}=\left\{x \in R: \mu_{A}(x)>0 \text { and } \lambda_{A}(x)<1\right\} \neq \phi
$$

is a sub-hemiring of $R$.

Proof. Let $x, y \in R_{(0,1)}$. Then $\mu_{A}(x)>0$ and $\lambda_{A}(x)<1, \mu_{A}(y)>0$ and $\lambda_{A}(y)<1$. Assume that $\mu_{A}(x+y)=0$ or $\lambda_{A}(x+y)=1$. If $\alpha \in\left\{\in, \in \vee q_{k}\right\}$, then, $x\left(\mu_{A}(x), \lambda_{A}(x)\right) \alpha A$ and $y\left(\mu_{A}(y), \lambda_{A}(y)\right) \alpha A$ but, $(x+y)\left(\min \left\{\mu_{A}(x), \mu_{A}(y)\right\}\right.$, $\left.\max \left\{\lambda_{A}(x), \lambda_{A}(y)\right\}\right) \bar{\beta} A$, for every $\beta \in\left\{\in, q_{k}, \in \vee q_{k}, \in \wedge q_{k}\right\}$, a contradiction. Also $x(1,0) q_{k} A$ and $y(1,0) q_{k} A$ but $(x+y)(1,0) \bar{\beta} A$ for every $\beta \in\left\{\in, q_{k}, \in \vee q_{k}, \in \wedge q_{k}\right\}$, a contradiction. Thus $\mu_{A}(x+y)>0$ and $\lambda_{A}(x+y)<1$. Therefore, $x+y \in R_{(0,1)}$.

Let $x, y \in R_{(0,1)}$. Then $\mu_{A}(x)>0$ and $\lambda_{A}(x)<1, \mu_{A}(y)>0$ and $\lambda_{A}(y)<1$. Suppose that $\mu_{A}(x y)=0$ or $\lambda_{A}(x y)=1$. If $\alpha \in\left\{\in, \in \vee q_{k}\right\}$, then $x\left(\mu_{A}(x), \lambda_{A}(x)\right) \alpha A \quad$ and $y\left(\mu_{A}(y), \lambda_{A}(y)\right) \alpha A \quad$ but, $(x y)\left(\min \left\{\mu_{A}(x), \mu_{A}(y)\right\}, \max \left\{\lambda_{A}(x), \lambda_{A}(y)\right\}\right) \bar{\beta} A$ for every $\beta \in\left\{\in, q_{k}, \in \vee q_{k}, \in \wedge q_{k}\right\}$, a contradiction. Also $x(1,0) q_{k} A$ and $y(1,0) q_{k} A$ but $(x y)(1,0) \bar{\beta} A$ for every $\beta \in\left\{\in, q_{k}, \in \vee q_{k}, \in \wedge q_{k}\right\}$, a contradiction. Thus $\mu_{A}(x y)>0$ and $\lambda_{A}(x y)<1$. Therefore, $x y \in R_{(0,1)}$. This completes the proof.

\section{$4\left(\in, \in \vee q_{k}\right)^{*}$-intuitionistic fuzzy ideals}

Definition 3. An IFS $A=\left(\mu_{A}, \lambda_{A}\right)$ of a hemiring $R$ is called an $\left(\in, \in \vee q_{k}\right)$-intuitionistic fuzzy sub-hemiring of $R$, if $\forall$ $x, y \in R$ and $t_{1}, t_{2} \in(0,1], s_{1}, s_{2} \in[0,1)$,

(1a) $x\left(t_{1}, s_{1}\right), y\left(t_{2}, s_{2}\right) \in A \Rightarrow(x+y)\left(\min \left(t_{1}, t_{2}\right), \max \left(s_{1}, s_{2}\right)\right) \in \vee q_{k} A$.

(2a) $x\left(t_{1}, s_{1}\right), y\left(t_{2}, s_{2}\right) \in A \Rightarrow(x y)\left(\min \left(t_{1}, t_{2}\right), \max \left(s_{1}, s_{2}\right)\right) \in \vee q_{k} A$.

Definition 4. An IFS A $=\left(\mu_{A}, \lambda_{A}\right)$ of a hemiring $R$ is called an $\left(\epsilon, \in \vee q_{k}\right)$-intuitionistic fuzzy left (right) ideal of $R$, if $\forall$ $x, y \in R$ and $t_{1}, t_{2} \in(0,1], s_{1}, s_{2} \in[0,1)$,

(1a) $x\left(t_{1}, s_{1}\right), y\left(t_{2}, s_{2}\right) \in A \Rightarrow(x+y)\left(\min \left(t_{1}, t_{2}\right), \max \left(s_{1}, s_{2}\right)\right) \in \vee q_{k} A$.

(3a) $y\left(t_{1}, s_{1}\right) \in A, x \in R \Rightarrow(x y)\left(t_{1}, s_{1}\right) \in \vee q_{k} A\left((y x)\left(t_{1}, s_{1}\right) \in \vee q_{k} A\right)$.

An IFS $A=\left(\mu_{A}, \lambda_{A}\right)$ of a hemiring $R$ is called an $\left(\in, \in \vee q_{k}\right)$-intuitionistic fuzzy ideal of $R$, if it is both $\left(\in, \in \vee q_{k}\right)$ intuitionistic fuzzy left ideal and $\left(\in, \in \vee q_{k}\right)$-intuitionistic fuzzy right ideal of $R$.

Theorem 5. Let $A$ be an intuitionistic fuzzy subset of a hemiring $R$. Then $(1 a) \Longrightarrow(1 b),(2 a) \Longrightarrow(2 b),(3 a) \Longrightarrow(3 b)$, where $\forall x, y \in R$ and $k \in[0,1)$, 
(1b) $\mu_{A}(x+y) \geq \min \left\{\mu_{A}(x), \mu_{A}(y), \frac{1-k}{2}\right\}$ and $\lambda_{A}(x+y) \leq \max \left\{\lambda_{A}(x), \lambda_{A}(y), \frac{1-k}{2}\right\}$.

(2b) $\mu_{A}(x y) \geq \min \left\{\mu_{A}(x), \mu_{A}(y), \frac{1-k}{2}\right\}$ and $\lambda_{A}(x y) \leq \max \left\{\lambda_{A}(x), \lambda_{A}(y), \frac{1-k}{2}\right\}$.

(3b) $\mu_{A}(x y) \geq \min \left\{\mu_{A}(y), \frac{1-k}{2}\right\}$ and $\lambda_{A}(x y) \leq \max \left\{\lambda_{A}(y), \frac{1-k}{2}\right\}$.

Proof. $(1 a) \Rightarrow(1 b)$ Let $A$ be an intuitionistic fuzzy subset of a hemiring $R$, and $(1 a)$ holds. Suppose that $(1 b)$ doesn't hold then there exist $x, y \in R$ such that $\mu_{A}(x+y)<\min \left\{\mu_{A}(x), \mu_{A}(y), \frac{1-k}{2}\right\}$ or $\lambda_{A}(x+y)>\max \left\{\lambda_{A}(x), \lambda_{A}(y), \frac{1-k}{2}\right\}$. So there exits three possible cases.

(i) $\mu_{A}(x+y)<\min \left\{\mu_{A}(x), \mu_{A}(y), \frac{1-k}{2}\right\}$ and $\lambda_{A}(x+y) \leq \max \left\{\lambda_{A}(x), \lambda_{A}(y), \frac{1-k}{2}\right\}$,

(ii) $\mu_{A}(x+y) \geq \min \left\{\mu_{A}(x), \mu_{A}(y), \frac{1-k}{2}\right\}$ and $\lambda_{A}(x+y)>\max \left\{\lambda_{A}(x), \lambda_{A}(y), \frac{1-k}{2}\right\}$,

(iii) $\mu_{A}(x+y)<\min \left\{\mu_{A}(x), \mu_{A}(y), \frac{1-k}{2}\right\}$ and $\lambda_{A}(x+y)>\max \left\{\lambda_{A}(x), \lambda_{A}(y), \frac{1-k}{2}\right\}$.

For the first case, there exist $t \in(0,1]$ such that $\mu_{A}(x+y)<t<\min \left\{\mu_{A}(x), \mu_{A}(y), \frac{1-k}{2}\right\}$. Now choose $s=1-t$, then clearly $x(t, s) \in A$ and $y(t, s) \in A$ but $(x+y)(t, s) \overline{\in \vee q_{k}} A$. Which is a contradiction. Second case is similar to this case.

Now consider case (iii), i.e $\mu_{A}(x+y)<\min \left\{\mu_{A}(x), \mu_{A}(y), \frac{1-k}{2}\right\}$ and $\lambda_{A}(x+y)>\max \left\{\lambda_{A}(x), \lambda_{A}(y), \frac{1-k}{2}\right\}$. Then there exist $t \in(0,1]$ and $s \in[0,1)$, such that $\mu_{A}(x+y)<t \leq \min \left\{\mu_{A}(x), \mu_{A}(y), \frac{1-k}{2}\right\}$ and $\lambda_{A}(x+y)>s \geq \max \left\{\lambda_{A}(x), \lambda_{A}(y), \frac{1-k}{2}\right\}$

$\Longrightarrow x(t, s) \in A$ and $y(t, s) \in A$ but $(x+y)(t, s) \overline{\in \vee q_{k}} A$. Which is again a contradiction. So our supposition is wrong. Hence $(1 b)$ holds.

Similarly we can prove $(2 a) \Longrightarrow(2 b),(3 a) \Longrightarrow(3 b)$.

Definition 5. Let $A=\left(\mu_{A}, \lambda_{A}\right)$ be an IFS of a hemiring $R$. Then $A$ is an $\left(\in, \in \vee q_{k}\right)^{*}$-intuitionistic fuzzy sub-hemiring of $R$ if it satisfies the conditions $(1 b)$ and $(2 b)$.

Definition 6. Let $A=\left(\mu_{A}, \lambda_{A}\right)$ be an IFS of a hemiring $R$. Then $A$ is an $\left(\in, \in \vee q_{k}\right)^{*}$-intuitionistic fuzzy left ideal of $R$ if it satisfies the conditions $(1 b)$ and $(3 b)$.

Remark. Every $\left(\in, \in \vee q_{k}\right)^{*}$-intuitionistic fuzzy left ideal (right ideal, sub-hemiring) $A=\left(\mu_{A}, \lambda_{A}\right)$ of $R$ need not be an $(\epsilon$, $\in \vee q_{k}$ )-intuitionistic fuzzy left ideal (right ideal, sub-hemiring) of $R$.

Example 1. Let $\mathbb{N}$ be the set of all non negative integers and $A=\left\langle\mu_{A}, \lambda_{A}\right\rangle$ be an IFS of $\mathbb{N}$ defined as follows:

$\mu_{A}(x)=\left\{\begin{array}{c}1 \quad \text { if } x=0 \\ 0.5 \quad \text { if } 1 \leq x \leq 4, \\ 0.4 \text { if } 4<x\end{array} \quad \lambda_{A}(x)=\left\{\begin{array}{cc}0 & \text { if } x=0 \\ 0.5 & \text { if } 1 \leq x \leq 4 \\ 0.4 & \text { if } 4<x\end{array}\right.\right.$

For all $x, y \in R$,

(1) $\mu_{A}(x+y) \geq \min \left\{\mu_{A}(x), \mu_{A}(y), 0.4\right\}$ and $\lambda_{A}(x+y) \leq \max \left\{\lambda_{A}(x), \lambda_{A}(y), 0.4\right\}$,

(2) $\mu_{A}(x y) \geq \min \left\{\mu_{A}(y), 0.4\right\}$ and $\lambda_{A}(x y) \leq \max \left\{\lambda_{A}(y), 0.4\right\}$,

(3) $\mu_{A}(x y) \geq \min \left\{\mu_{A}(x), 0.4\right\}$ and $\lambda_{A}(x y) \leq \max \left\{\lambda_{A}(x), 0.4\right\}$.

Thus $A=\left(\mu_{A}, \lambda_{A}\right)$ is an $\left(\in, \in \vee q_{0.2}\right)^{*}$-intuitionistic fuzzy ideal of $\mathbb{N}$. But $2(0.45,0.55), 3(0.45,0.55) \in A \Longrightarrow$ (2.3) $(0.45,0.55) \overline{\in \vee q_{0.2}} A$. Thus $A=\left(\mu_{A}, \lambda_{A}\right)$ is not an $\left(\in, \in \vee q_{0.2}\right)$-intuitionistic fuzzy ideal of $\mathbb{N}$.

Definition 7. For any intuitionistic fuzzy set $A=\left(\mu_{A}, \lambda_{A}\right)$ in $R$ and $t \in(0,1], s \in[0,1)$ and $k \in[0,1)$ we define $U_{(t, s)}=\{x \in R: x(t, s) \in A\}, A_{(t, s)_{k}}=\left\{x \in R: x(t, s) q_{k} A\right\}$ and $[A]_{(t, s)_{k}}=\left\{x \in R: x(t, s) \in \vee q_{k} A\right\}$. 
Obviously, $[A]_{(t, s)_{k}}=A_{(t, s)_{k}} \cup U_{(t, s)}$, where $U_{(t, s)}, A_{(t, s)_{k}}$ and $[A]_{(t, s)_{k}}$ are called $\in$-level set, $q_{k}$-level set and $\in \vee q_{k}$-level set of $A=\left(\mu_{A}, \lambda_{A}\right)$, respectively.

Lemma 1. Every intuitionistic fuzzy subset $A=\left(\mu_{A}, \lambda_{A}\right)$ of a hemiring $R$ satisfies the following condition:

$t \in\left(0, \frac{1-k}{2}\right], s \in\left[\frac{1-k}{2}, 1\right) \Longrightarrow[A]_{(t, s)_{k}}=U_{(t, s)}$

Proof. Let $t \in\left(0, \frac{1-k}{2}\right]$, and $s \in\left[\frac{1-k}{2}, 1\right)$. It is clear that $U_{(t, s)} \subseteq[A]_{(t, s)_{k}}$. Let $x \in[A]_{(t, s)_{k}}$. If $x \notin U_{(t, s)}$, then $\mu_{A}(x)<t$, or $\lambda_{A}(x)>s$ and so $\mu_{A}(x)+t<2 t \leq 1-k$, or $\lambda_{A}(x)+s>2 s \geq 1-k$. This shows that $x(t, s) \overline{q_{k}} A$.i.e., $x \notin A_{(t, s))_{k}}$ and thus $x \notin U_{(t, s)} \cup A_{(t, s)_{k}}=[A]_{(t, s)_{k}}$. This is a contradiction. Thus $x \in U_{(t, s)}$. Therefore $[A]_{(t, s)_{k}} \subseteq U_{(t, s)}$.

Theorem 6. If $A$ is an $\left(\in, \in \vee q_{k}\right)^{*}$-intuitionistic fuzzy ideal of $R$, then the set $A_{(t, s)_{k}}$ is an ideal of $R$ when it is non-empty for all $t \in\left(\frac{1-k}{2}, 1\right], s \in\left[0, \frac{1-k}{2}\right)$.

Proof. Assume that $A$ is an $\left(\in, \in \vee q_{k}\right)^{*}$-intuitionistic fuzzy ideal of $R$, and let $t \in\left(\frac{1-k}{2}, 1\right], s \in\left[0, \frac{1-k}{2}\right)$ be such that $A_{(t, s)_{k}} \neq \phi$. Let $x, y \in A_{(t, s)_{k}}$. Then $\mu_{A}(x)+t+k>1, \lambda_{A}(x)+s+k<1$ and $\mu_{A}(y)+t+k>1, \lambda_{A}(y)+s+k<1$. As $\mu_{A}(x+y) \geq \min \left\{\mu_{A}(x), \mu_{A}(y), \frac{1-k}{2}\right\}, \lambda_{A}(x+y) \leq \max \left\{\lambda_{A}(x), \lambda_{A}(y), \frac{1-k}{2}\right\}$. We have $\mu_{A}(x+y) \geq \min \left\{1-t-k, \frac{1-k}{2}\right\}$, $\lambda_{A}(x+y) \leq \max \left\{1-s-k, \frac{1-k}{2}\right\}$. Since $t \in\left(\frac{1-k}{2}, 1\right]$, and $s \in\left[0, \frac{1-k}{2}\right)$, so $1-t-k<\frac{1-k}{2}$ and $1-s-k>\frac{1-k}{2}$, thus $\mu_{A}(x+y)>1-t-k$ and $\lambda_{A}(x+y)<1-s-k$. Hence $x+y \in A_{(t, s)_{k}}$. Let $x \in A_{(t, s)_{k}}$ and $y \in R$. Then $\mu_{A}(x)+t+k>1$, $\lambda_{A}(x)+s+k<1$. Then $\mu_{A}(x)>1-t-k, \lambda_{A}(x)<1-s-k$. Since $A$ is an $(\in, \in \vee q)^{*}$-intuitionistic fuzzy ideal of $R$, we have $\mu_{A}(x y) \geq \min \left\{\mu_{A}(x), \frac{1-k}{2}\right\}, \lambda_{A}(x+y) \leq \max \left\{\lambda_{A}(x), \frac{1-k}{2}\right\}$. Implies that $\mu_{A}(x y) \geq \min \left\{1-t-k, \frac{1-k}{2}\right\}, \lambda_{A}(x y) \leq$ $\max \left\{1-s-k, \frac{1-k}{2}\right\}$. Since $t \in\left(\frac{1-k}{2}, 1\right]$, and $s \in\left[0, \frac{1-k}{2}\right)$, so $1-t-k<\frac{1-k}{2}$ and $1-s-k>\frac{1-k}{2}$, thus $\mu_{A}(x y)>1-t-k$ and $\lambda_{A}(x y)<1-s-k$. This implies $x y \in A_{(t, s)}$. Similarly $x y \in A_{(t, s)_{k}}$. Hence $A_{(t, s)_{k}}$ is an ideal of $R$.

Theorem 7. For any intuitionistic fuzzy subset A of $R$, the following are equivalent:

(i) A is an $\left(\in, \in \vee q_{k}\right)^{*}$-intuitionistic fuzzy ideal of $R$.

(ii) For all $t \in\left(0, \frac{1-k}{2}\right]$, and $s \in\left[\frac{1-k}{2}, 1\right), U_{(t, s)} \neq \phi \Longrightarrow U_{(t, s)}$ is an ideal of $R$.

Proof. Let $A$ be an $\left(\in, \in \vee q_{k}\right)^{*}$-intuitionistic fuzzy ideal of $R$ and $x, y \in U_{(t, s)}$ for some $t \in\left(0, \frac{1-k}{2}\right], s \in\left[\frac{1-k}{2}, 1\right)$. Then $\mu_{A}(x+y) \geq \min \left\{\mu_{A}(x), \mu_{A}(y), \frac{1-k}{2}\right\} \geq \min \left\{t, \frac{1-k}{2}\right\}=t$ and $\lambda_{A}(x+y) \leq \max \left\{\lambda_{A}(x), \lambda_{A}(y), \frac{1-k}{2}\right\} \leq \max \left\{s, \frac{1-k}{2}\right\}=s$, which implies $x+y \in U_{(t, s)}$. Now, if $x \in U_{(t, s)}$ and $y \in R$ then $\mu_{A}(x y) \geq \min \left\{\mu_{A}(x), \frac{1-k}{2}\right\} \geq \min \left\{t, \frac{1-k}{2}\right\}=t$ and $\lambda_{A}(x y) \leq \max \left\{\lambda_{A}(x), \frac{1-k}{2}\right\} \leq \max \left\{s, \frac{1-k}{2}\right\}=s$, which implies $x y \in U_{(t, s)}$. Similarly $y x \in U_{(t, s)}$. This shows that $U_{(t, s)}$ is an ideal of $R$.

Conversely, assume that for every $t \in\left(0, \frac{1-k}{2}\right]$, and $s \in\left[\frac{1-k}{2}, 1\right)$, each non-empty $U_{(t, s)}$ is an ideal of $R$. Suppose $A$ is not an $\left(\in, \in \vee q_{k}\right)^{*}$-intuitionistic fuzzy ideal of $R$, then there exist $x, y \in R$ such that one of the following three cases is true.

(i) $\mu_{A}(x+y)<\min \left\{\mu_{A}(x), \mu_{A}(y), \frac{1-k}{2}\right\}$ and $\lambda_{A}(x+y) \leq \max \left\{\lambda_{A}(x), \lambda_{A}(y), \frac{1-k}{2}\right\}$.

(ii) $\mu_{A}(x+y) \geq \min \left\{\mu_{A}(x), \mu_{A}(y), \frac{1-k}{2}\right\}$ and $\lambda_{A}(x+y)>\max \left\{\lambda_{A}(x), \lambda_{A}(y), \frac{1-k}{2}\right\}$.

(iii) $\mu_{A}(x+y)<\min \left\{\mu_{A}(x), \mu_{A}(y), \frac{1-k}{2}\right\}$ and $\lambda_{A}(x+y)>\max \left\{\lambda_{A}(x), \lambda_{A}(y), \frac{1-k}{2}\right\}$.

For the first case, $t \in\left(0, \frac{1-k}{2}\right]$ such that $\mu_{A}(x+y)<t \leq \min \left\{\mu_{A}(x), \mu_{A}(y), \frac{1-k}{2}\right\}$. Now choose $s=1-t$, then clearly $x$ ,$y \in U_{(t, s)}$ but $x+y \notin U_{(t, s)}$. Which is a contradiction. Case $(i i)$ is similar to the case $(i)$.

Now consider case (iii), then there exist $t \in\left(0, \frac{1-k}{2}\right]$, and $s \in\left[\frac{1-k}{2}, 1\right)$, such that $\mu_{A}(x+y)<t \leq \min \left\{\mu_{A}(x), \mu_{A}(y), \frac{1-k}{2}\right\}$ and $\lambda_{A}(x+y)>s \geq \max \left\{\lambda_{A}(x), \lambda_{A}(y), \frac{1-k}{2}\right\} \Longrightarrow x, y \in U_{(t, s)}$ but $x+y \notin U_{(t, s)}$. Which is a contradiction. So our supposition is wrong, hence $\mu_{A}(x+y) \geq \min \left\{\mu_{A}(x), \mu_{A}(y), \frac{1-k}{2}\right\}$ and $\lambda_{A}(x+y) \leq \max \left\{\lambda_{A}(x), \lambda_{A}(y), \frac{1-k}{2}\right\}$ for all 
$x, y \in R$.

In a similar way we can show that $\mu_{A}(x y) \geq \min \left\{\mu_{A}(x), \frac{1-k}{2}\right\}$ and $\lambda_{A}(x y) \leq \max \left\{\lambda_{A}(x), \frac{1-k}{2}\right\}$, $\mu_{A}(x y) \geq \min \left\{\mu_{A}(y), \frac{1-k}{2}\right\}$ and $\lambda_{A}(x y) \leq \max \left\{\lambda_{A}(y), \frac{1-k}{2}\right\}$ for all $x, y \in R$.

Theorem 8. Let $\left\{A_{i}: i \in I\right\}$ be a family of $\left(\in, \in \vee q_{k}\right)^{*}$-intuitionistic fuzzy sub-hemiring of $R$. Then $A=\cap_{i \in I} A_{i}$ is an $(\in$, $\left.\in \vee q_{k}\right)^{*}$-intuitionistic fuzzy sub-hemiring of $R$.

Proof. Straightforward.

Theorem 9. Let $\left\{A_{i}: i \in I\right\}$ be a family of $\left(\in, \in \vee q_{k}\right)^{*}$-intuitionistic fuzzy left (right) ideals of $R$. Then $A=\cap_{i \in I} A_{i}$ is an $\left(\in, \in \vee q_{k}\right)^{*}$-intuitionistic fuzzy left (right) ideal of $R$.

Proof. Straightforward.

\section{Regular and idempotent hemirings}

Definition 8. Let $A$ and $B$ be two intuitionistic fuzzy subsets of a hemiring $R$, then $A{ }^{\circ} B$ is defined as, $A \cdot{ }_{k} B=\left\langle\mu_{A} \cdot{ }_{k} \mu_{B}, \lambda_{A} \cdot{ }_{k} \lambda_{B}\right\rangle$ where

$$
\begin{aligned}
& \left(\mu_{A}{ }_{k} \mu_{B}\right)(x)=\left\{\begin{array}{l}
\bigvee_{x=\sum_{i=1}^{p} y_{i} z_{i}}\left[\bigwedge_{1 \leq i \leq p}\left[\mu_{A}\left(y_{i}\right) \wedge \mu_{B}\left(z_{i}\right)\right]\right] \wedge \frac{1-k}{2} \\
0 \quad \text { if } x \text { cannot be expressed as } x=\sum_{i=1}^{p} y_{i} z_{i}
\end{array}\right. \\
& \left(\lambda_{A}{ }_{k} \lambda_{B}\right)(x)=\left\{\begin{array}{l}
\bigwedge_{x=\sum_{i=1}^{p} y_{i} z_{i}}\left[\bigvee_{1 \leq i \leq p}^{\bigvee}\left[\lambda_{A}\left(y_{i}\right) \vee \lambda_{B}\left(z_{i}\right)\right]\right] \vee \frac{1-k}{2} \\
1 \text { if } x \text { cannot be expressed as } x=\sum_{i=1}^{p} y_{i} z_{i}
\end{array}\right.
\end{aligned}
$$

where $x \in R$.

Definition 9. let $A$ and $B$ an intuitionistic fuzzy subsets of $R$. We define the intuitionistic fuzzy subsets $A_{k}, A \cap_{k} B, A \cup_{k} B$ and $A \cdot{ }_{k} B$ of $R$ as follows:

$$
\begin{aligned}
& A_{k}=\left(\mu_{A} \wedge \frac{1-k}{2}, \lambda_{B} \vee \frac{1-k}{2}\right), \\
& A \cap_{k} B=(A \cap B)_{k}=\left(\mu_{A} \wedge_{k} \mu_{B}, \lambda_{A} \vee_{k} \lambda_{B}\right), \\
& A \cup_{k} B=(A \cup B)_{k}=\left(\mu_{A} \vee_{k} \mu_{B}, \lambda_{A} \wedge_{k} \lambda_{B}\right) .
\end{aligned}
$$

Theorem 10. Let $A$ be an $\left(\in, \in \vee q_{k}\right)^{*}$-intuitionistic fuzzy sub-hemiring of $R$. Then $A_{k}$ is an $\left(\in, \in \vee q_{k}\right)^{*}$-intuitionistic fuzzy sub-hemiring of $R$.

Proof. Suppose $A$ is an $\left(\in, \in \vee q_{k}\right)^{*}$-intuitionistic fuzzy sub-hemiring of $R$ and $x, y \in R$. Then

$$
\begin{aligned}
\left(\mu_{A} \wedge \frac{1-k}{2}\right)(x+y) & =\mu_{A}(x+y) \wedge \frac{1-k}{2} \\
& \geq\left(\min \left\{\mu_{A}(x), \mu_{A}(y), \frac{1-k}{2}\right\}\right) \wedge \frac{1-k}{2} \\
& =\min \left\{\mu_{A}(x) \wedge \frac{1-k}{2}, \mu_{A}(y) \wedge \frac{1-k}{2}, \frac{1-k}{2}\right\} \\
& =\min \left\{\left(\mu_{A} \wedge \frac{1-k}{2}\right)(x),\left(\mu_{A} \wedge \frac{1-k}{2}\right)(y), \frac{1-k}{2}\right\},
\end{aligned}
$$


and

$$
\begin{aligned}
\left(\lambda_{A} \vee \frac{1-k}{2}\right)(x+y) & =\lambda_{A}(x+y) \vee \frac{1-k}{2} \\
& \leq\left(\max \left\{\lambda_{A}(x), \lambda_{A}(y), \frac{1-k}{2}\right\}\right) \vee \frac{1-k}{2} \\
& =\max \left\{\lambda_{A}(x) \vee \frac{1-k}{2}, \lambda_{A}(y) \vee \frac{1-k}{2}, \frac{1-k}{2}\right\} \\
& =\max \left\{\left(\lambda_{A} \vee \frac{1-k}{2}\right)(x),\left(\lambda_{A}(y) \vee \frac{1-k}{2}\right)(y), \frac{1-k}{2}\right\}
\end{aligned}
$$

Similarly we can show that

$$
\left(\mu_{A} \wedge \frac{1-k}{2}\right)(x y) \geq \min \left\{\left(\mu_{A} \wedge \frac{1-k}{2}\right)(x),\left(\mu_{A} \wedge \frac{1-k}{2}\right)(y), \frac{1-k}{2}\right\}
$$

and

$$
\left(\lambda_{A} \vee \frac{1-k}{2}\right)(x y) \leq \max \left\{\left(\lambda_{A} \vee \frac{1-k}{2}\right)(x),\left(\lambda_{A}(y) \vee \frac{1-k}{2}\right)(y), \frac{1-k}{2}\right\} .
$$

This shows that $A_{k}=A \cap \frac{1-k}{2}$ is an $\left(\in, \in \vee q_{k}\right)^{*}$-intuitionistic fuzzy sub-hemiring of $R$.

Theorem 11. Let $A$ be an $\left(\in, \in \vee q_{k}\right)^{*}$-intuitionistic fuzzy ideal of $R$. Then $A_{k}$ is an $\left(\in, \in \vee q_{k}\right)^{*}$-intuitionistic fuzzy ideal of $R$.

Proof. This proof is similar to the proof of the theorem 10,

Remark. let $A$ and $B$ be intuitionistic fuzzy subsets of $R$.Then the following hold.

(i) $A \cap_{k} B=\left(A_{k} \cap B_{k}\right)$.

(ii) $A \cup_{k} B=\left(A_{k} \cup B_{k}\right)$.

(iii) $A \cdot{ }_{k} B=\left(A_{k} \cdot B_{k}\right)$.

Proof. let $x \in R$,

(1) $\left(\mu_{A} \wedge_{k} \mu_{B}\right)(x)=\left(\mu_{A} \wedge \mu_{B}\right)(x) \wedge \frac{1-k}{2}=\mu_{A}(x) \wedge \mu_{B}(x) \wedge \frac{1-k}{2}=\left(\mu_{A}(x) \wedge \frac{1-k}{2}\right) \wedge\left(\mu_{B}(x) \wedge \frac{1-k}{2}\right)$

and

$$
=\mu_{A_{k}}(x) \wedge \mu_{B_{K}}(x)=\left(\mu_{A_{k}} \wedge \mu_{B_{K}}\right)(x)
$$

$$
\begin{aligned}
\left(\lambda_{A} \vee_{k} \lambda_{B}\right)(x) & \left.=\left(\lambda_{A} \vee \lambda_{B}\right)(x) \vee \frac{1-k}{2}\right)=\lambda_{A}(x) \vee \lambda_{B}(x) \vee \frac{1-k}{2}=\left(\lambda_{A}(x) \vee \frac{1-k}{2}\right) \vee\left(\lambda_{B}(x) \vee \frac{1-k}{2}\right) \\
& =\lambda_{A_{k}}(x) \vee \lambda_{B_{K}}(x)=\left(\lambda_{A_{k}} \vee \lambda_{B_{K}}\right)(x) .
\end{aligned}
$$

Hence (1) holds. Similarly we can prove (2).

(3) If $x$ is not expressible as $x=\sum_{i=1}^{p} y_{i} z_{i}$ where $y_{i}, z_{i} \in R$, then $\left(\mu_{A} \cdot \mu_{B}\right)(x)=0$.

Thus $\left(\mu_{A} \cdot k \mu_{B}\right)(x)=\left(\mu_{A} \cdot \mu_{B}\right)(x) \wedge \frac{1-k}{2}=0$. As $x$ is not expressible as $x=\sum_{i=1}^{p} y_{i} z_{i}$ so $\left(\mu_{A_{k}} \cdot \mu_{B_{k}}\right)(x)=0 \Longrightarrow \mu_{A}{ }_{k} \mu_{B}=\mu_{A_{k}} \cdot \mu_{B_{k}}$ and $\left(\lambda_{A} \cdot \lambda_{B}\right)(x)=1$, thus $\left(\lambda_{A}{ }_{k} \lambda_{B}\right)(x)=\left(\lambda_{A} \cdot \lambda_{B}\right)(x) \vee \frac{1-k}{2}=1$ as $x$ is not expressible as $x=\sum_{i=1}^{p} y_{i} z_{i}$ so $\left(\lambda_{A_{k}} \cdot \lambda_{B_{k}}\right)(x)=1 \Longrightarrow \lambda_{A}{ }^{\cdot k} \lambda_{B}=\lambda_{A_{k}} \cdot \lambda_{B_{k}}$. Hence (3) holds.

Theorem 12. If $A$ and $B$ are $\left(\in, \in \vee q_{k}\right)^{*}$-intuitionistic fuzzy ideals of $R$ then $A \cdot{ }_{k} B$ is an $\left(\in, \in \vee q_{k}\right)^{*}$-intuitionistic fuzzy ideal of $R$.

Proof. Let $x, y \in R$ be such that $x=\sum_{i=1}^{p} a_{i} b_{i}$, and $y=\sum_{j=1}^{q} a_{i}^{\prime} b_{i}^{\prime}$. Then

$$
\left(\mu_{A}{ }_{k} \mu_{B}\right)(x)=\bigvee_{x=\sum_{i=1}^{p} a_{i} b_{i}}\left[\bigwedge_{1 \leq i \leq p}\left[\mu_{A}\left(a_{i}\right) \wedge \mu_{B}\left(b_{i}\right)\right]\right] \wedge \frac{1-k}{2}
$$


and

$$
\left(\mu_{A} \cdot k \mu_{B}\right)\left(x^{\prime}\right)=\bigvee_{x^{\prime}=\sum_{j=1}^{q} a_{i}^{\prime} b_{i}^{\prime}}\left[\bigwedge_{1 \leq i \leq p}\left[\mu_{A}\left(a_{i}^{\prime}\right) \wedge \mu_{B}\left(b_{i}^{\prime}\right)\right]\right] \wedge \frac{1-k}{2}
$$

Thus

$$
\begin{aligned}
& \left(\mu_{A} \cdot{ }_{k} \mu_{B}\right)(x) \wedge\left(\mu_{A} \cdot{ }_{k} \mu_{B}\right)\left(x^{\prime}\right) \wedge \frac{1-k}{2}=\left\{\begin{array}{c}
{\left[\bigvee_{x=\sum_{i=1}^{p} a_{i} b_{i}}\left[\wedge_{1 \leq i \leq p}\left[\mu_{A}\left(a_{i}\right) \wedge \mu_{B}\left(b_{i}\right)\right]\right] \wedge \frac{1-k}{2}\right] \wedge} \\
{\left[\bigvee_{x^{\prime}=\sum_{j=1}^{q} a_{i}^{\prime} b_{i}^{\prime}}\left[\wedge_{1 \leq i \leq p}\left[\mu_{A}\left(a_{i}^{\prime}\right) \wedge \mu_{B}\left(b_{i}^{\prime}\right)\right]\right] \wedge \frac{1-k}{2}\right] \wedge \frac{1-k}{2}}
\end{array}\right. \\
& =\left[\bigvee_{x=\sum_{i=1}^{p} a_{i} b_{i} x^{\prime}=\sum_{j=1}^{q} a_{i}^{\prime} b_{i}^{\prime}}\left[\begin{array}{c}
{\left[\bigwedge_{1 \leq i \leq p}\left[\mu_{A}\left(a_{i}\right) \wedge \mu_{B}\left(b_{i}\right)\right]\right] \wedge} \\
{\left[\bigwedge_{1 \leq j \leq q}\left[\mu_{A}\left(a_{i}^{\prime}\right) \wedge \mu_{B}\left(b_{i}^{\prime}\right)\right]\right]}
\end{array}\right] \wedge \frac{1-k}{2}\right] \\
& \leq\left[\bigvee_{x+x^{\prime}=\sum_{k=1}^{s} a^{\prime \prime} b^{\prime \prime}}\left[\bigwedge_{1 \leq k \leq s}\left[\mu_{A}\left(a^{\prime \prime}\right) \wedge \mu_{B}\left(b^{\prime \prime}\right)\right]\right] \wedge \frac{1-k}{2}\right] \\
& =\left(\mu_{A} \cdot{ }_{k} \mu_{B}\right)\left(x+x^{\prime}\right)
\end{aligned}
$$

and

$$
\begin{aligned}
& \left(\lambda_{A} \cdot{ }_{k} \lambda_{B}\right)(x)=\left[\bigwedge_{x=\sum_{i=1}^{p} a_{i} b_{i}}\left[\bigvee_{1 \leq i \leq p}\left[\lambda_{A}\left(a_{i}\right) \vee \lambda_{B}\left(b_{i}\right)\right]\right] \vee \frac{1-k}{2}\right], \\
& \left(\lambda_{A} \cdot{ }_{k} \lambda_{B}\right)\left(x^{\prime}\right)=\left[\bigwedge_{x^{\prime}=\sum_{j=1}^{q} a_{i}^{\prime} b_{i}^{\prime}}\left[\bigvee_{1 \leq i \leq p}\left[\lambda_{A}\left(a_{i}^{\prime}\right) \vee \lambda_{B}\left(b_{i}^{\prime}\right)\right]\right] \vee \frac{1-k}{2}\right] .
\end{aligned}
$$

Thus

$$
\begin{aligned}
\left(\lambda_{A} \cdot{ }_{k} \lambda_{B}\right)(x) \vee\left(\lambda_{A} \cdot{ }_{k} \lambda_{B}\right)\left(x^{\prime}\right) \vee \frac{1-k}{2}=\left\{\begin{array}{l}
{\left[\bigwedge_{x=\sum_{i=1}^{p} a_{i} b_{i}}\left[\bigvee_{1 \leq i \leq p}\left[\lambda_{A}\left(a_{i}\right) \vee \lambda_{B}\left(b_{i}\right)\right]\right] \vee \frac{1-k}{2}\right] \vee} \\
{\left[\bigwedge_{x^{\prime}=\sum_{j=1}^{q} a_{i}^{\prime} b_{i}^{\prime}}\left[\bigvee_{1 \leq i \leq p}\left[\lambda_{A}\left(a_{i}^{\prime}\right) \vee \lambda_{B}\left(b_{i}^{\prime}\right)\right]\right] \vee \frac{1-k}{2}\right] \vee \frac{1-k}{2}}
\end{array}\right. \\
=\left[\begin{array}{c}
\left.\bigwedge_{x=\sum_{i=1}^{p} a_{i} b_{i} x^{\prime}=\sum_{j=1}^{q} a_{i}^{\prime} b_{i}^{\prime}}\left[\begin{array}{c}
\left.\left[\bigvee_{1 \leq i \leq p}\left[\lambda_{A}\left(a_{i}\right) \vee \lambda_{B}\left(b_{i}\right)\right]\right] \wedge\right] \vee \\
{\left[\bigvee_{1 \leq j \leq q}\left[\lambda_{A}\left(a_{i}^{\prime}\right) \vee \lambda_{B}\left(b_{i}^{\prime}\right)\right]\right]}
\end{array}\right] \vee \frac{1-k}{2}\right]
\end{array}\right.
\end{aligned}
$$

$\geq\left[\bigwedge_{x+x^{\prime}=\sum_{k=1}^{s} a^{\prime \prime} b^{\prime \prime}}\left[\bigvee_{1 \leq k \leq s}\left[\lambda_{A}\left(a^{\prime \prime}\right) \vee \lambda_{B}\left(b^{\prime \prime}\right)\right]\right] \vee \frac{1-k}{2}\right]$

$=\left(\lambda_{A} \cdot{ }_{k} \lambda_{B}\right)\left(x+x^{\prime}\right)$

$\Longrightarrow\left\{\left(\lambda_{A}{ }^{\cdot k} \lambda_{B}\right)(x) \vee\left(\lambda_{A}{ }_{k} \lambda_{B}\right)\left(x^{\prime}\right) \vee \frac{1-k}{2}\right\} \geq\left(\lambda_{A} \cdot{ }_{k} \lambda_{B}\right)\left(x+x^{\prime}\right)$. Also, $\left(\mu_{A} \cdot{ }_{k} \mu_{B}\right)(x) \wedge \frac{1-k}{2}$

$=\left[\left[\bigvee_{x=\sum_{i=1}^{p} a_{i} b_{i}}\left[\wedge_{1 \leq i \leq p}\left[\mu_{A}\left(a_{i}\right) \wedge \mu_{B}\left(b_{i}\right)\right]\right]\right] \wedge \frac{1-k}{2}\right] \frac{1-k}{2}$

$=\left[\bigvee_{x=\sum_{i=1}^{p} a_{i} b_{i}}\left[\bigwedge_{1 \leq i \leq p}\left[\mu_{A}\left(a_{i}\right) \wedge \mu_{B}\left(b_{i}\right) \frac{1-k}{2}\right]\right]\right] \wedge \frac{1-k}{2}$

$\leq\left[\bigvee_{x=\sum_{i=1}^{p} a_{i} b_{i}}\left[\bigwedge_{1 \leq i \leq p}\left[\mu_{A}\left(a_{i}\right) \wedge \mu_{B}\left(b_{i} r\right)\right]\right]\right] \wedge \frac{1-k}{2}$ 
$\leq\left[\bigvee_{x r=\sum_{j=1}^{q} a_{i}^{\prime} b_{i}^{\prime}}\left[\Lambda_{1 \leq j \leq q}\left[\mu_{A}\left(a_{i}^{\prime}\right) \wedge \mu_{B}\left(b_{i}^{\prime}\right)\right]\right]\right] \wedge \frac{1-k}{2}$

$=\left(\mu_{A}{ }_{k} \mu_{B}\right)(x r)$.

Thus $\left\{\left(\mu_{A} \cdot k \mu_{B}\right)(x) \wedge \frac{1-k}{2}\right\} \leq\left(\mu_{A} \cdot k \mu_{B}\right)(x r)$.

Similarly we can prove $\left(\lambda_{A}{ }^{\cdot} \lambda_{B}\right)(x r) \leq\left\{\left(\lambda_{A}{ }_{k} \lambda_{B}\right)(x) \vee \frac{1-k}{2}\right\} \Longrightarrow A \cdot{ }_{k} B$ is an $\left(\in, \in \vee q_{k}\right)^{*}$-intuitionistic fuzzy right ideal of $R$. On the same line it can be proved that $\left\{\left(\mu_{A}{ }_{k} \mu_{B}\right)(x) \wedge \frac{1-k}{2}\right\} \leq\left(\mu_{A} \cdot_{k} \mu_{B}\right)(r x)$ and $\left(\lambda_{A} \cdot{ }_{k} \lambda_{B}\right)(r x) \leq\left\{\left(\lambda_{A} \cdot{ }_{k} \lambda_{B}\right)(x r) \vee \frac{1-k}{2}\right\}$. Thus $A \cdot{ }_{k} B$ is an $\left(\in, \in \vee q_{k}\right)^{*}$-intuitionistic fuzzy ideal of $R$.

Theorem 13. If $A$ and $B$ are $(\in, \in \vee q)^{*}$-intuitionistic fuzzy left(right) ideals of $R$, then so is $A \cap_{k} B$.

Proof. We only consider the case of $\left(\in, \in \vee q_{k}\right)^{*}$-intuitionistic fuzzy left ideals.

Let $x, y \in R$. Then

$$
\begin{aligned}
\left(\mu_{A} \wedge_{k} \mu_{B}\right)(x+y) & =\min \left\{\mu_{A}(x+y), \mu_{B}(x+y), \frac{1-k}{2}\right\} \\
& \geq \min \left\{\min \left\{\mu_{A}(x), \mu_{A}(y), \frac{1-k}{2}\right\}, \min \left\{\mu_{B}(y), \mu_{B}(x), \frac{1-k}{2}\right\}, \frac{1-k}{2}\right\} \\
& =\min \left\{\min \left\{\mu_{A}(x), \mu_{B}(x), \frac{1-k}{2}\right\}, \min \left\{\mu_{A}(y), \mu_{B}(y), \frac{1-k}{2}\right\}, \frac{1-k}{2}\right\} \\
& =\min \left\{\left(\mu_{A} \wedge_{k} \mu_{B}\right)(x),\left(\mu_{A} \wedge_{k} \mu_{B}\right)(y), \frac{1-k}{2}\right\}
\end{aligned}
$$

and

$$
\begin{aligned}
\left(\lambda_{A} \vee_{k} \lambda_{B}\right)(x+y) & =\max \left\{\lambda_{A}(x+y), \lambda_{B}(x+y), \frac{1-k}{2}\right\} \\
& \leq \max \left\{\max \left\{\lambda_{A}(x), \lambda_{A}(y), \frac{1-k}{2}\right\}, \max \left\{\lambda_{B}(x), \lambda_{B}(y), \frac{1-k}{2}\right\}, \frac{1-k}{2}\right\} \\
& =\max \left\{\max \left\{\lambda_{A}(x), \lambda_{B}(x), \frac{1-k}{2}\right\}, \max \left\{\lambda_{A}(y), \lambda_{B}(y), \frac{1-k}{2}\right\}, \frac{1-k}{2}\right\} \\
& =\max \left\{\left(\lambda_{A} \vee_{k} \lambda_{B}\right)(x),\left(\lambda_{A} \vee_{k} \lambda_{B}\right)(y), \frac{1-k}{2}\right\}
\end{aligned}
$$

Now

$$
\begin{aligned}
\left(\mu_{A} \wedge_{k} \mu_{B}\right)(x . y) & =\min \left\{\mu_{A}(x . y), \mu_{B}(x \cdot y), \frac{1-k}{2}\right\} \\
& \geq \min \left\{\min \left\{\mu_{A}(y), \frac{1-k}{2}\right\}, \min \left\{\mu_{B}(y), \frac{1-k}{2}\right\}, \frac{1-k}{2}\right\} \\
& =\min \left\{\min \left\{\mu_{A}(y), \mu_{B}(y), \frac{1-k}{2}\right\}, \frac{1-k}{2}\right\}=\min \left\{\left(\mu_{A} \wedge_{k} \mu_{B}\right)(y), \frac{1-k}{2}\right\}
\end{aligned}
$$


and

$$
\begin{aligned}
\left(\lambda_{A} \vee_{k} \lambda_{B}\right)(x \cdot y) & =\max \left\{\lambda_{A}(x \cdot y), \lambda_{B}(x . y), \frac{1-k}{2}\right\} \\
& \leq \max \left\{\max \left\{\lambda_{A}(y), \frac{1-k}{2}\right\}, \max \left\{\lambda_{B}(y), \frac{1-k}{2}\right\}, \frac{1-k}{2}\right\} \\
& =\max \left\{\max \left\{\lambda_{A}(y), \lambda_{B}(y), \frac{1-k}{2}\right\}, \frac{1-k}{2}\right\}=\max \left\{\left(\lambda_{A} \vee_{k} \lambda_{B}\right)(y), \frac{1-k}{2}\right\}
\end{aligned}
$$

Thus $A \cap_{k} B$ is an $\left(\in, \in \vee q_{k}\right)^{*}$-intuitionistic fuzzy left ideal of $R$.

Theorem 14. If $A$ is an $\left(\in, \in \vee q_{k}\right)^{*}$-intuitionistic fuzzy right ideal, and $B$ is an $\left(\in, \in \vee q_{k}\right)^{*}$-intuitionistic fuzzy left ideal of $R$, then $A \cdot{ }_{k} B \subseteq A \cap_{k} B$.

Proof. Let $A$ and $B$ be $\left(\in, \in \vee q_{k}\right)^{*}$-intuitionistic fuzzy right and left ideals of $R$ respectively. For any $x \in R$,

$$
\begin{aligned}
& \left(\mu_{A} \cdot k \mu_{B}\right)(x)=\bigvee_{x=\sum_{i=1}^{p} a_{i} b_{i}}\left[\bigwedge_{1 \leq i \leq p}\left[\mu_{A}\left(a_{i}\right) \wedge \mu_{B}\left(b_{i}\right)\right]\right] \wedge \frac{1-k}{2} \\
& =\bigvee_{x=\sum_{i=1}^{p} a_{i} b_{i}}\left[\bigwedge_{1 \leq i \leq p}\left[\mu_{A}\left(a_{i}\right) \wedge \frac{1-k}{2}\right] \wedge\left[\mu_{B}\left(b_{i}\right) \wedge \frac{1-k}{2}\right]\right] \wedge \frac{1-k}{2} \\
& \leq \bigvee_{x=\sum_{i=1}^{p} a_{i} b_{i}}\left[\bigwedge_{1 \leq i \leq p}\left[\mu_{A}\left(a_{i} b_{i}\right) \wedge \mu_{B}\left(a_{i} b_{i}\right)\right]\right] \wedge \frac{1-k}{2} \\
& =\bigvee_{x=\sum_{i=1}^{p} a_{i} b_{i}}\left[\left(\bigwedge_{1 \leq i \leq p} \mu_{A}\left(a_{i} b_{i}\right)\right) \wedge\left(\bigwedge_{1 \leq i \leq p} \mu_{B}\left(a_{i} b_{i}\right)\right)\right] \wedge \frac{1-k}{2} \\
& \leq\left[\bigvee_{x=\sum_{i=1}^{p} a_{i} b_{i}}\left[\mu_{A}(x) \wedge \mu_{B}(x)\right]\right] \wedge \frac{1-k}{2}=\left(\mu_{A} \wedge_{k} \mu_{B}\right)(x),
\end{aligned}
$$

and

$$
\begin{aligned}
\left(\lambda_{A} \cdot{ }_{k} \lambda_{B}\right)(x) & =\bigwedge_{x=\sum_{i=1}^{p} a_{i} b_{i}}\left[\bigvee_{1 \leq i \leq p}\left[\lambda_{A}\left(a_{i}\right) \vee \lambda_{B}\left(b_{i}\right)\right]\right] \vee \frac{1-k}{2} \\
& =\bigwedge_{x=\sum_{i=1}^{p} a_{i} b_{i}}\left[\bigvee_{1 \leq i \leq p}\left[\lambda_{A}\left(a_{i}\right) \vee \frac{1-k}{2}\right] \vee\left[\lambda_{B}\left(b_{i}\right) \vee \frac{1-k}{2}\right]\right] \vee \frac{1-k}{2} \\
& \geq \bigwedge_{x=\sum_{i=1}^{p} a_{i} b_{i}}\left[\bigvee_{1 \leq i \leq p}\left[\lambda_{A}\left(a_{i} b_{i}\right) \vee \lambda_{B}\left(a_{i} b_{i}\right)\right]\right] \vee \frac{1-k}{2} \\
& =\bigwedge_{x=\sum_{i=1}^{p} a_{i} b_{i}}\left[\left(\bigvee_{1 \leq i \leq p}^{\bigvee} \lambda_{A}\left(a_{i} b_{i}\right)\right) \vee\left(\bigvee_{1 \leq i \leq p} \lambda_{B}\left(a_{i} b_{i}\right)\right)\right] \vee \frac{1-k}{2} \\
& \geq \bigwedge_{x=\sum_{i=1}^{p} a_{i} b_{i}}\left[\lambda_{A}(x) \vee \lambda_{B}(x)\right] \vee \frac{1-k}{2}=\left(\lambda_{A} \vee{ }_{k} \lambda_{B}\right)(x)
\end{aligned}
$$

Thus $A \cdot{ }_{k} B \subseteq A \cap_{k} B$. 
Definition 10. Let $A$ and $B$ be $\left(\in, \in \vee q_{k}\right)^{*}$-intuitionistic fuzzy ideals of $R$. The intuitionistic fuzzy subset $A+{ }_{k} B$ of $R$ is defined by

$$
A+{ }_{k} B=\left(\mu_{A}+{ }_{k} \mu_{B}, \lambda_{A}+{ }_{k} \lambda_{B}\right)
$$

where

$$
\begin{gathered}
\left(\mu_{A}+{ }_{k} \mu_{B}\right)(x)=\bigvee_{x=y+z}\left[\mu_{A}(y) \wedge \mu_{B}(z)\right] \wedge \frac{1-k}{2}, \\
\left(\lambda_{A}+{ }_{k} \lambda_{B}\right)(x)=\bigwedge_{x=y+z}\left[\lambda_{A}(y) \vee \lambda_{B}(z)\right] \vee \frac{1-k}{2} \text { for } x \in R .
\end{gathered}
$$

Proposition 1. For $\left(\in, \in \vee q_{k}\right)^{*}$-intuitionistic fuzzy ideals $A$ and $B$ of $R, A+{ }_{k} B$ is an $\left(\in, \in \vee q_{k}\right)^{*}$-intuitionistic fuzzy ideal of $R$.

Proof. For any $x, x^{\prime} \in R$,

$$
\begin{aligned}
& \left(\mu_{A}+{ }_{k} \mu_{B}\right)(x) \wedge\left(\mu_{A}+{ }_{k} \mu_{B}\right)\left(x^{\prime}\right) \wedge \frac{1-k}{2}=\left[\begin{array}{c}
{\left[\bigvee_{x=y+z}\left[\mu_{A}(y) \wedge \mu_{B}(z)\right] \wedge \frac{1-k}{2}\right] \wedge} \\
{\left[\bigvee_{x^{\prime}=y^{\prime}+z^{\prime}}\left[\mu_{A}\left(y^{\prime}\right) \wedge \mu_{B}\left(z^{\prime}\right)\right] \wedge \frac{1-k}{2}\right] \wedge \frac{1-k}{2}}
\end{array}\right] \\
& =\left[\bigvee_{x=y+z x^{\prime}=y^{\prime}+z^{\prime}}\left[\begin{array}{l}
\left.\left[\left[\mu_{A}(y) \wedge \mu_{B}(z)\right] \wedge \frac{1-k}{2}\right] \wedge\right] \\
\left.\left[\left[\mu_{A}\left(y^{\prime}\right) \wedge \mu_{B}\left(z^{\prime}\right)\right] \wedge \frac{1-k}{2}\right]\right]
\end{array}\right] \wedge \frac{1-k}{2}\right. \\
& =\left[\bigvee_{x=y+z} \bigvee_{x^{\prime}=y^{\prime}+z^{\prime}}\left[\begin{array}{c}
\left.\left[\left[\mu_{A}(y) \wedge \mu_{A}\left(y^{\prime}\right)\right] \wedge \frac{1-k}{2}\right] \wedge\right] \\
{\left[\left[\mu_{B}(z) \wedge \mu_{B}\left(z^{\prime}\right)\right] \wedge \frac{1-k}{2}\right]}
\end{array}\right]\right) \wedge \frac{1-k}{2} \\
& \leq \bigvee_{x=y+z x^{\prime}=y^{\prime}+z^{\prime}}\left[\mu_{A}\left(y+y^{\prime}\right) \wedge \mu_{B}\left(z+z^{\prime}\right)\right] \wedge \frac{1-k}{2} \\
& \leq\left(\mu_{A}+{ }_{k} \mu_{B}\right)\left(x+{ }_{k} x^{\prime}\right),
\end{aligned}
$$

and

$$
\begin{aligned}
\left(\lambda_{A}+{ }_{k} \lambda_{B}\right)(x) \vee\left(\lambda_{A}+{ }_{k} \lambda_{B}\right)\left(x^{\prime}\right) \vee \frac{1-k}{2} & =\left[\begin{array}{c}
{\left[\bigwedge_{x=y+z}\left[\lambda_{A}(y) \vee \lambda_{B}(z)\right] \vee \frac{1-k}{2}\right] \vee} \\
\left.\bigwedge_{x^{\prime}=y^{\prime}+z^{\prime}}\left[\lambda_{A}\left(y^{\prime}\right) \vee \lambda_{B}\left(z^{\prime}\right)\right] \vee \frac{1-k}{2}\right] \vee \frac{1-k}{2}
\end{array}\right] \\
& =\left[\bigwedge_{x=y+z} \bigwedge_{x^{\prime}=y^{\prime}+z^{\prime}}\left[\begin{array}{c}
{\left[\left[\lambda_{A}(y) \vee \lambda_{B}(z)\right] \vee \frac{1-k}{2}\right] \vee} \\
\left.\left[\lambda_{A}\left(y^{\prime}\right) \vee \lambda_{B}\left(z^{\prime}\right)\right] \vee \frac{1-k}{2}\right]
\end{array}\right]\right] \vee \frac{1-k}{2} \\
& =\left[\bigwedge_{x=y+z} \bigwedge_{x^{\prime}=y^{\prime}+z^{\prime}}\left[\begin{array}{c}
{\left[\left[\lambda_{A}(y) \vee \lambda_{A}\left(y^{\prime}\right)\right] \vee \frac{1-k}{2}\right] \vee} \\
\left.\left[\lambda_{B}(z) \vee \lambda_{B}\left(z^{\prime}\right)\right] \vee \frac{1-k}{2}\right]
\end{array}\right]\right] \vee \frac{1-k}{2} \\
& \geq \bigwedge_{x=y+z x^{\prime}=y^{\prime}+z^{\prime}}\left[\lambda_{A}\left(y+y^{\prime}\right) \vee \lambda_{B}\left(z+z^{\prime}\right)\right] \vee \frac{1-k}{2} \\
& \geq\left(\lambda_{A}+{ }_{k} \lambda_{B}\right)\left(x+{ }_{k} x^{\prime}\right)
\end{aligned}
$$


Again,

$$
\begin{aligned}
\left(\mu_{A}+{ }_{k} \mu_{B}\right)(x) \wedge \frac{1-k}{2} & =\left[\bigvee_{x=y+z}\left[\mu_{A}(y) \wedge \mu_{B}(z)\right] \wedge \frac{1-k}{2}\right] \wedge \frac{1-k}{2} \\
& =\left[\bigvee_{x=y+z}\left[\left(\mu_{A}(y) \wedge \frac{1-k}{2}\right) \wedge\left(\mu_{B}(z) \wedge \frac{1-k}{2}\right)\right]\right] \\
& \leq\left[\bigvee_{x=y+z}\left[\mu_{A}(y a) \wedge \mu_{B}(z a)\right]\right] \wedge \frac{1-k}{2} \\
& \leq\left[\bigvee_{x a=y^{\prime}+z^{\prime}}\left[\mu_{A}\left(y^{\prime}\right) \wedge \mu_{B}\left(z^{\prime}\right)\right]\right] \wedge \frac{1-k}{2}=\left(\mu_{A}+{ }_{k} \mu_{B}\right)(x a)
\end{aligned}
$$

and

$$
\begin{aligned}
\left(\lambda_{A}+{ }_{k} \lambda_{B}\right)(x) \vee \frac{1-k}{2} & =\left[\bigwedge_{x=y+z}\left[\lambda_{A}(y) \vee \lambda_{B}(z)\right] \vee \frac{1-k}{2}\right] \vee \frac{1-k}{2} \\
& =\left[\bigwedge_{x=y+z}\left[\left(\lambda_{A}(y) \vee \frac{1-k}{2}\right) \vee\left(\lambda_{B}(z)\right) \vee \frac{1-k}{2}\right]\right] \vee \frac{1-k}{2} \\
& \geq\left[\bigwedge_{x=y+z}\left(\lambda_{A}(y a) \vee \lambda_{B}(z a)\right)\right] \vee \frac{1-k}{2} \\
& \geq\left[\bigwedge_{x a=y^{\prime}+z^{\prime}}\left(\lambda_{A}\left(y^{\prime}\right) \vee \lambda_{B}\left(z^{\prime}\right)\right)\right] \vee \frac{1-k}{2}=\left(\lambda_{A}+{ }_{k} \lambda_{B}\right)(x a) .
\end{aligned}
$$

Similarly we can prove

$$
\left(\mu_{A}+{ }_{k} \mu_{B}\right)(x) \wedge \frac{1-k}{2} \leq\left(\mu_{A}+{ }_{k} \mu_{B}\right)(a x) \text { and }\left(\lambda_{A}+{ }_{k} \lambda_{B}\right)(x) \vee \frac{1-k}{2} \geq\left(\lambda_{A}+{ }_{k} \lambda_{B}\right)(a x) .
$$

Hence $A+{ }_{k} B$ is an $\left(\in, \in \vee q_{k}\right)^{*}$-intuitionistic fuzzy ideal of $R$.

Definition 11.[18] If $S \subseteq R$, then intuitionistic characteristic function of $S$ is denoted by $C_{S}=\left(\chi_{S}, \chi_{S}^{c}\right)$ and is defined by

$\chi_{S}(x)=\left\{\begin{array}{ll}1 & \text { if } x \in S \\ 0 & \text { if } x \notin S\end{array}\right.$ and $\chi_{S}^{c}(x)=\left\{\begin{array}{ll}0 & \text { if } x \in S \\ 1 & \text { if } x \notin S\end{array}\right.$ In particular, we let $\overline{1}=\left(\chi_{R}, \chi_{R}^{c}\right)$ be the intuitionistic fuzzy set in $R$.

Lemma 2. A non-empty subset $L$ of a hemiring $R$ is a left ideal of $R$ if and only if the intuitionistic characteristic function $C_{L}=\left(\chi_{L}, \chi_{L}^{c}\right)$ is an $\left(\in, \in \vee q_{k}\right)^{*}$-intuitionistic fuzzy left ideal of $R$.

Proof. Let $L$ be a left ideal of $R$, then obviously $C_{L}$ is an $\left(\in, \in \vee q_{k}\right)^{*}$-intuitionistic fuzzy left ideal of $R$.

Conversely assume that $C_{L}$ is an $\left(\in, \in \vee q_{k}\right)^{*}$-intuitionistic fuzzy left ideal of $R$. Let $x, y \in L$. Then $\chi_{L}(x)=1, \chi_{L}^{c}(x)=0$, and $\chi_{L}(y)=1, \chi_{L}^{c}(y)=0$ so $x(1,0), y(1,0) \in C_{L}$. Since $C_{L}$ is an $\left(\in, \in \vee q_{k}\right)^{*}$-intuitionistic fuzzy left ideal, so $\left(\chi_{L}\right)(x+y) \geq \min \left\{\chi_{L}(x), \chi_{L}(y), \frac{1-k}{2}\right\}$ and $\left(\chi_{L}^{c}\right)(x+y) \leq \max \left\{\chi_{L}^{c}(x), \chi_{L}^{c}(y), \frac{1-k}{2}\right\}$ i.e $\left(\chi_{L}\right)(x+y)=1$ and $\left(\chi_{L}^{c}\right)(x+y)=0$. Thus $x+y \in L$.

Let $y \in L$ and $x \in R$. Then $\chi_{L}(y)=1$, and $\chi_{L}(y)=0$ so $y(1,0) \in C_{L}$. Since $C_{L}$ is an $\left(\in, \in \vee q_{k}\right)^{*}$-intuitionistic fuzzy left 
ideal, so $\left(\chi_{L}\right)(x y) \geq \min \left\{\chi_{L}(y), \frac{1-k}{2}\right\}$ and $\left(\chi_{L}^{c}\right)(x y) \leq \max \left\{\chi_{L}^{c}(y), \frac{1-k}{2}\right\}$. i.e. $\left(\chi_{L}\right)(x y)=1$ and $\left(\chi_{L}^{c}\right)(x y)=0$. Hence $x y \in L$. Thus $L$ is a left ideal of $R$

Lemma 3. A non-empty subset $L$ of a hemiring $R$ is a left ideal of $R$ if and only if the intuitionistic fuzzy set $\left(C_{L}\right)_{k}=$ $\left(\chi_{L} \wedge \frac{1-k}{2}, \chi_{L}^{c} \vee \frac{1-k}{2}\right)$ is an $\left(\in, \in \vee q_{k}\right)^{*}$-intuitionistic fuzzy left ideal of $R$.

Proof.Straightforward.

Lemma 4. Let $A$ and $B$ be non-empty subsets of a hemiring $R$. Then the following hold:

(1) $C_{A} \cap{ }_{k} C_{B}=\left(C_{A \cap B}\right)_{k}$

(2) $C_{A} \cdot{ }_{k} C_{B}=\left(C_{A \cdot B}\right)_{k}$.

Proof.Straightforward.

Theorem 15. For a hemiring $R$, the following conditions are equivalent:

(i) $R$ is hemiregular.

(ii) $A \cap_{k} B=A \cdot{ }_{k} B$ for every $\left(\in, \in \vee q_{k}\right)^{*}$-intuitionistic fuzzy right ideal $A$ and every $\left(\in, \in \vee q_{k}\right)^{*}$-intuitionistic fuzzy left ideal $B$ of $R$.

Proof. Let $A$ be an $\left(\in, \in \vee q_{k}\right)^{*}$-intuitionistic fuzzy right ideal and $B$ be an $\left(\in, \in \vee q_{k}\right)^{*}$-intuitionistic fuzzy left ideal of $R$ and $x \in R$. Then there exists $a \in R$, such that $x=x a x$. Now

$$
\begin{array}{r}
\left(\mu_{A} \cdot_{k} \mu_{B}\right)(x)=\left\{\bigvee_{x=\sum_{i=1}^{p} y_{i} z_{i}}\left[\bigwedge_{1 \leq i \leq p}\left[\mu_{A}\left(y_{i}\right) \wedge \mu_{B}\left(z_{i}\right)\right]\right] \wedge \frac{1-k}{2}\right\} \\
\geq\left[\mu_{A}(x a) \wedge \mu_{B}(x) \wedge \frac{1-k}{2}\right] \\
\geq\left[\mu_{A}(x) \wedge \mu_{B}(x) \wedge \frac{1-k}{2}\right]=\left(\mu_{A} \wedge_{k} \mu_{B}\right)(x)
\end{array}
$$

and

$$
\begin{aligned}
\left(\lambda_{A} \cdot{ }_{k} \lambda_{B}\right)(x)=\left\{\bigwedge_{x=\sum_{i=1}^{p} y_{i} z_{i}}\left[\bigvee_{1 \leq i \leq p}\left[\lambda_{A}\left(y_{i}\right) \vee \lambda_{B}\left(z_{i}\right)\right]\right] \vee \frac{1-k}{2}\right\} & \leq\left[\lambda_{A}(x a) \vee \lambda_{B}(x) \vee \frac{1-k}{2}\right] \\
& \leq\left[\lambda_{A}(x) \vee \lambda_{B}(x) \vee \frac{1-k}{2}\right]=\left(\lambda_{A} \vee \lambda_{B}\right)(x)
\end{aligned}
$$

Thus $A \cap_{k} B \subseteq A \cdot{ }_{k} B$.

By Theorem $14 A \cdot{ }_{k} B \subseteq A \cap_{k} B$. Hence $A \cdot{ }_{k} B=A \cap_{k} B$.

$(i i) \Longrightarrow(i)$ Let $A$ and $B$ be right ideal and left ideal of $R$ respectively. Then $C_{A}$ is an $\left(\in, \in \vee q_{k}\right)^{*}$-intuitionistic fuzzy right ideal and $C_{B}$ is an $\left(\in, \in \vee q_{k}\right)^{*}$-intuitionistic fuzzy left ideal of $R$, by assumption

$$
C_{A} \cdot{ }_{k} C_{B}=C_{A} \cap{ }_{k} C_{B} \Longrightarrow\left(C_{A} \cdot C_{B}\right)_{k}=\left(C_{A} \cap C_{B}\right)_{k} \Longrightarrow\left(C_{A B}\right)_{k}=\left(C_{A \cap B}\right)_{k} \Longrightarrow A B=A \cap B .
$$

Thus by Theorem $1 R$ is regular.

Theorem 16. The following assertions for a hemiring $R$ with identity are equivalent:

(1) $R$ is fully idempotent. 
(2) Each $\left(\in, \in \vee q_{k}\right)^{*}$-intuitionistic fuzzy ideal of $R$ is idempotent. (an $\left(\in, \in \vee q_{k}\right)^{*}$-intuitionistic fuzzy ideal A of $R$ is called idempotent if $A \cdot{ }_{k} A=A_{k}$.)

(3) for each pair of $\left(\in, \in \vee q_{k}\right)^{*}$-intuitionistic fuzzy ideals $A$ and $B$ of $R, A \cap_{k} B=A \cdot_{k} B$.

(4) If $R$ is assumed to be commutative, then the above assertions are equivalent to $R$ is regular.

Proof. (1) $\Longrightarrow(2)$. Let $A=\left(\mu_{A}, \lambda_{A}\right)$ be an $\left(\in, \in \vee q_{k}\right)^{*}$-intuitionistic fuzzy ideal of $R$. For any $x \in R$, by Theorem 14 $A \cdot{ }_{k} A \subseteq A_{k}$.

Since each ideal of $R$ is idempotent, therefore, $(x)=(x)^{2}$ for each $x \in R$. Since $x \in(x)$ it follows that $x \in(x)^{2}=R x R R x R$. Hence $x=\sum_{i=1}^{q} a_{i} x a_{i}^{\prime} b_{i} x b_{i}^{\prime}$ and $q \in N$. Now,

$$
\begin{aligned}
\left(\mu_{A} \wedge \frac{1-k}{2}\right)(x)=\mu_{A}(x) \wedge \mu_{A}(x) \wedge \frac{1-k}{2} & =\left[\mu_{A}(x) \wedge \frac{1-k}{2}\right] \wedge\left[\mu_{A}(x) \wedge \frac{1-k}{2}\right] \wedge \frac{1-k}{2} \\
& \leq \mu_{A}\left(a_{i} x a_{i}^{\prime}\right) \wedge \mu_{A}\left(b_{i} x b_{i}^{\prime}\right) \wedge \frac{1-k}{2},(1 \leq i \leq q)
\end{aligned}
$$

Therefore,

$$
\begin{aligned}
\left(\mu_{A} \wedge \frac{1-k}{2}\right)(x) & \leq \bigwedge_{1 \leq i \leq q}\left[\mu_{A}\left(a_{i} x a_{i}^{\prime}\right) \wedge \mu_{A}\left(b_{i} x b_{i}^{\prime}\right)\right] \wedge \frac{1-k}{2} \\
& \leq \bigvee_{x=\Sigma_{i=1}^{q} a_{i} x a_{i}^{\prime} b_{i} x b_{i}^{\prime}}\left[\bigwedge_{1 \leq i \leq q}\left[\mu_{A}\left(a_{i} x a_{i}^{\prime}\right) \wedge \mu_{A}\left(b_{i} x b_{i}^{\prime}\right)\right]\right] \wedge \frac{1-k}{2} \\
& \leq \bigvee_{x=\sum_{j=1}^{r} a_{j} b_{j}}\left[\bigwedge_{1 \leq j \leq r}\left[\mu_{A}\left(a_{j}\right) \wedge \mu_{A}\left(b_{j}\right)\right]\right] \wedge \frac{1-k}{2}=\left(\mu_{A} \cdot k \mu_{A}\right)(x)
\end{aligned}
$$

and

$$
\begin{aligned}
\left(\lambda_{A} \vee \frac{1-k}{2}\right)(x) & =\lambda_{A}(x) \vee \lambda_{A}(x) \vee \frac{1-k}{2} \\
& =\left[\lambda_{A}(x) \vee \frac{1-k}{2}\right] \vee\left[\lambda_{A}(x) \vee \frac{1-k}{2}\right] \vee \frac{1-k}{2} \\
& \geq \lambda_{A}\left(a_{i} x a_{i}^{\prime}\right) \vee \lambda_{A}\left(b_{i} x b_{i}^{\prime}\right) \vee \frac{1-k}{2},(1 \leq i \leq q)
\end{aligned}
$$

Therefore,

$$
\begin{aligned}
\left(\lambda_{A} \vee \frac{1-k}{2}\right)(x) & \geq \bigvee_{1 \leq i \leq q}\left[\lambda_{A}\left(a_{i} x a_{i}^{\prime}\right) \vee \lambda_{A}\left(b_{i} x b_{i}^{\prime}\right)\right] \vee \frac{1-k}{2} \\
& \geq \bigwedge_{x=\sum_{i=1}^{q} a_{i} x a_{i}^{\prime} b_{i} x b_{i}^{\prime}}\left[\bigvee_{1 \leq i \leq q}\left[\lambda_{A}\left(a_{i} x a_{i}^{\prime}\right) \vee \lambda_{A}\left(b_{i} x b_{i}^{\prime}\right)\right]\right] \vee \frac{1-k}{2} \\
& \geq \bigwedge_{x=\sum_{j=1}^{r} a_{j} b_{j}}\left[\bigvee_{1 \leq j \leq r}\left[\lambda_{A}\left(a_{j}\right) \vee \lambda_{A}\left(b_{j}\right)\right]\right] \vee \frac{1-k}{2}=\left(\lambda_{A} \cdot{ }_{k} \lambda_{A}\right)(x)
\end{aligned}
$$

Thus $A \cdot{ }_{k} A=A_{k}$.

$(2) \Longrightarrow(1)$. Let $I$ be an ideal of $R$. Then $C_{I}$, the intuitionistic characteristic function of $I$, is an $\left(\in, \in \vee q_{k}\right)^{*}$-intuitionistic 
fuzzy ideal of $R$. Hence, $C_{I} \cdot{ }_{k} C_{I}=\left(C_{I} \cdot C_{I}\right)_{k}=\left(C_{I^{2}}\right)_{k}=\left(C_{I}\right)_{k}$. It follows that $I^{2}=I$.

$(1) \Longrightarrow(3)$. Let $A$ and $B$ be $\left(\in, \in \vee q_{k}\right)^{*}$-intuitionistic fuzzy ideals of $R$.

By Theorem $14 A \cdot{ }_{k} B \subseteq A \cap_{k} B$. Again since $R$ is fully idempotent, $(x)=(x)^{2}$, for any $x \in R$. Hence, as argued in the first part of the proof of this theorem, we have

$$
\begin{aligned}
&\left(\mu_{A} \wedge_{k} \mu_{B}\right)(x)=\left(\mu_{A}\right)(x) \wedge\left(\mu_{B}\right)(x) \wedge \frac{1-k}{2} \\
& \leq \bigvee_{x=\sum_{1=1}^{p} a_{i} b_{i}}\left[\bigwedge_{1 \leq i \leq r}\left[\mu_{A}\left(a_{i}\right) \wedge \mu_{B}\left(b_{i}\right)\right]\right] \wedge \frac{1-k}{2}=\left(\mu_{A} \cdot k\right. \\
&\left.\mu_{B}\right)(x)
\end{aligned}
$$

and

$$
\begin{aligned}
\left(\lambda_{A} \vee_{k} \lambda_{B}\right)(x) & =\lambda_{A}(x) \vee \lambda_{B}(x) \vee \frac{1-k}{2} \\
& \geq \bigwedge_{x=\sum_{i=1}^{p} a_{i} b_{i}}\left[\bigvee_{1 \leq i \leq r}\left[\lambda_{A}\left(a_{i}\right) \vee \lambda_{B}\left(b_{i}\right)\right]\right] \vee \frac{1-k}{2}=\left(\lambda_{A} \cdot{ }_{k} \lambda_{B}\right)(x)
\end{aligned}
$$

Thus $A \cdot{ }_{k} B=A \cap_{k} B$.

(3) $\Longrightarrow(1)$. Let $A$ and $B$ be any pair of $\left(\in, \in \vee q_{k}\right)^{*}$-intuitionistic fuzzy ideals of $R$. We have $A{ }^{\circ} B=A \cap_{k} B$. Take $A=B$. Thus $A \cdot{ }_{k} A=A \cap_{k} A=A_{k}$, where $A$ is any $\left(\in, \in \vee q_{k}\right)^{*}$-intuitionistic fuzzy ideal of $R$. Hence, (3) $\Longrightarrow(2)$. Since we already proved that (1) and (2) are equivalent,hence (3) $\Longrightarrow(1)$ and so (1) $\Leftrightarrow(3)$. This establishes $(1) \Leftrightarrow(2) \Leftrightarrow(3)$. Finally, If $A$ is commutative then it is easy to verify that (1) $\Leftrightarrow(4)$.

Theorem 17. For a hemiring $R$ with 1 , the following conditions are equivalent.

(1) $R$ is right weakly regular hemiring.

(2) All $\left(\in, \in \vee q_{k}\right)^{*}$-intuitionistic fuzzy right ideals of $R$ are idempotent.

(3) $A \cdot{ }_{k} B=A \cap_{k} B$ for $\left(\in, \in \vee q_{k}\right)^{*}$-intuitionistic fuzzy right ideal $A$ and all $\left(\in, \in \vee q_{k}\right)^{*}$-intuitionistic fuzzy two-sided ideals $B$ of $R$.

Proof. (1) $\Longrightarrow(2)$ Let $A$ be an $\left(\in, \in \vee q_{k}\right)^{*}$-intuitionistic fuzzy right ideal of $R$. Then we have $A{ }_{k} A \subseteq A_{k}$.

For the reverse inclusion, let $x \in R$. Since $R$ is right weakly regular, so there exist $a_{i}, b_{i} \in R$ such that $x=\sum_{i=i}^{q} x a_{i} x b_{i}$. Now we have

$$
\begin{aligned}
\left(\mu_{A} \wedge \frac{1-k}{2}\right)(x) & =\mu_{A}(x) \wedge \mu_{A}(x) \wedge \frac{1-k}{2} \\
& =\left[\mu_{A}(x) \wedge \frac{1-k}{2}\right] \wedge\left[\mu_{A}(x) \wedge \frac{1-k}{2}\right] \wedge \frac{1-k}{2} \\
& \leq \mu_{A}\left(x a_{i}\right) \wedge \mu_{A}\left(x b_{i}\right) \wedge \frac{1-k}{2},(1 \leq i \leq q)
\end{aligned}
$$


Therefore,

$$
\begin{aligned}
\left(\mu_{A} \wedge \frac{1-k}{2}\right)(x) & \leq \bigwedge_{1 \leq i \leq q}\left[\mu_{A}\left(x a_{i}\right) \wedge \mu_{A}\left(x b_{i}\right)\right] \wedge \frac{1-k}{2} \\
& \leq \bigvee_{x=\sum_{i=1}^{q} \times a_{i} x_{i}}\left[\bigwedge_{1 \leq i \leq q}\left[\mu_{A}\left(x a_{i}\right) \wedge \mu_{A}\left(x b_{i}\right)\right]\right] \wedge \frac{1-k}{2} \\
& \leq \bigvee_{x=\sum_{j=1}^{r} a_{j} b_{j}}\left[\bigwedge_{1 \leq j \leq r}\left[\mu_{A}\left(a_{j}\right) \wedge \mu_{A}\left(b_{j}\right)\right]\right] \wedge \frac{1-k}{2}=\left(\mu_{A} \cdot{ }_{k} \mu_{A}\right)(x)
\end{aligned}
$$

and

$$
\begin{aligned}
\left(\lambda_{A} \vee \frac{1-k}{2}\right)(x) & =\lambda_{A}(x) \vee \lambda_{A}(x) \vee \frac{1-k}{2} \\
& =\left[\lambda_{A}(x) \vee \frac{1-k}{2}\right] \vee\left[\lambda_{A}(x) \vee \frac{1-k}{2}\right] \vee \frac{1-k}{2} \\
& \geq \lambda_{A}\left(x a_{i}\right) \vee \lambda_{A}\left(x b_{i}\right) \vee \frac{1-k}{2},(1 \leq i \leq q) .
\end{aligned}
$$

Therefore,

$$
\begin{aligned}
\left(\lambda_{A} \vee \frac{1-k}{2}\right)(x) & \geq \bigvee_{1 \leq i \leq q}\left[\lambda_{A}\left(x a_{i}\right) \vee \lambda_{A}\left(x b_{i}\right)\right] \vee \frac{1-k}{2} \\
& \geq \bigwedge_{x=\Sigma_{i=1}^{q} x a_{i} x b_{i}}\left[\bigvee_{1 \leq i \leq q}\left[\lambda_{A}\left(x a_{i}\right) \vee \lambda_{A}\left(x b_{i}\right)\right]\right] \vee \frac{1-k}{2} \\
& \geq \bigwedge_{x=\Sigma_{j=1}^{r} a_{j} b_{j}}\left[\bigvee_{1 \leq j \leq r}\left[\lambda_{A}\left(a_{j}\right) \vee \lambda_{A}\left(b_{j}\right)\right]\right] \vee \frac{1-k}{2}=\left(\lambda_{A} \cdot{ }_{k} \lambda_{A}\right)(x)
\end{aligned}
$$

Thus $A \cdot{ }_{k} A=A_{k}$

(2) $\Longrightarrow$ (3) Let $A$ and $B$ be $\left(\in, \in \vee q_{k}\right)^{*}$-intuitionistic fuzzy right ideal and $\left(\in, \in \vee q_{k}\right)^{*}$-intuitionistic fuzzy two-sided ideal of $R$ respectively. Then $A \cap_{k} B$ is an $\left(\in, \in \vee q_{k}\right)^{*}$-intuitionistic fuzzy right ideal of $R$. By Theorem 14 $A \cdot{ }_{k} B \subseteq A \cap_{k} B$. By hypothesis,

$$
\left(A \cap_{k} B\right)=\left(A \cap_{k} B\right) \cdot{ }_{k}\left(A \cap_{k} B\right) \subseteq A \cdot{ }_{k} B
$$

Hence $A \cdot{ }_{k} B=A \cap_{k} B$.

(3) $\Longrightarrow$ (1) Let $B$ be a right ideal of $R$ and $A$ be two sided-ideal of $R$. Then the intuitionistic characteristic function $C_{A}$ and $C_{B}$ are $\left(\in, \in \vee q_{k}\right)^{*}$-intuitionistic fuzzy two-sided ideal and $\left(\in, \in \vee q_{k}\right)^{*}$-intuitionistic fuzzy right ideal of $R$, respectively. Hence by hypothesis

$$
C_{B}{ }_{k} C_{A}=C_{B} \cap_{k} C_{A} \Longrightarrow\left(C_{B \cdot A}\right)_{k}=\left(C_{A \cap B}\right)_{k} \Longrightarrow B \cdot A=B \cap A .
$$

Thus by Theorem $2, R$ is right weakly regular hemiring. 


\section{References}

[1] J. Ahsan, fully Idempotent Semirings, Proc. Japan Acad. 69, Ser. A (1993), 185-188.

[2] J. Ahsan, Semirings Characterized by Their Fuzzy Ideals, J. Fuzzy Math. 6 (1998), 181-192.

[3] J. Ahsan, K. Saifullah, M. F. Khan, Fuzzy semirings, Fuzzy Sets and Systems 60 (1993) 309-320.

[4] K. Atanassov; Intuitionistic fuzzy sets, Fuzzy Sets and Systems, 20 (1986) 87-96.

[5] S.K. Bhakat, P. Das, $(\in, \in \vee q)$-fuzzy subgroups, Fuzzy Sets and Systems 80 (1996) 359-368.

[6] D. Coker, M. Demirci, On intuitionistic fuzzy points, Notes IFS 1 (2) (1995) 79-84.

[7] W.A. Dudek, Special types of intuitionistic fuzzy left h-ideals of hemirings, Soft Comput. 12 (2008) 359-364.

[8] W.A. Dudek, M. Shabir, I. Ali, $(\alpha, \beta)$-fuzzy ideals of hemirings, Comput. Math. Appl. 58 (2) (2009) 310-321.

[9] J. S. Golan, Semirings and their Applications, Kluwer Acad. Publ. , 1999

[10] U. Hebisch, H.J. Weinert, Semirings: Algebraic Theory and Applications in the Computer Science, World Scientific, 1998.

[11] A. Hussain, M. Shabir, Soft Finite State Machine, Journal of Intelligent and Fuzzy System, 2015, ( Accepted).

[12] A. Hussain, M. Shabir, Cubic Finite State Machine, Annals of Fuzzy Mathematics and Informatics, 2015, ( Accepted).

[13] Y.B. Jun, Generalization of $(\in, \in \vee q)$-fuzzy subalgebras in BCK/BCI-algebras, Comput. Math. Appl. 58 (2009) 1383-1390.

[14] Y.B. Jun, On $(\phi, \psi)$-intuitionistic fuzzy fubgroups, KYUNGPOOK Math. J. 45 (2005) 87-94.

[15] Y.B. Jun, W.A. Dudek, M. Shabir, Generalizations of $(\alpha, \beta)$-fuzzy ideals of hemirings.

[16] A. Khan, M. Shabir, $(\alpha, \beta)$-fuzzy interior ideals in ordered semigroups, Lobachevskii J. Math. 30 (2009) 30-39.

[17] A. Khan, Y.B Jun, N.H. Sarmin, F.M. Khan, Ordered semigroups characterized by $\left(\in, \in \vee q_{k}\right)$-fuzzy generalized bi-ideals, Neural Comput \& Applic (2011).

[18] V.N. Mishra, Some Problems on Approximations of Functions in Banach Spaces, Ph.D. Thesis (2007), Indian Institute of Technology, Roorkee - 247 667, Uttarakhand, India.

[19] J.N. Mordeson, D.S. Malik, Fuzzy Automata and Languages, Theory and Applications, in: Computational Mathematics Series, Chapman and Hall/CRC, Boca Raton, 2002.

[20] V. Murali, Fuzzy points of equivalent fuzzy subsets, Information Science 158 (2004) 277-288.

[21] P.M. Pu, Y.M. Liu, Fuzzy topology I, neighborhood structure of a fuzzy point and Moore-Smith convergence, J. Math. Anal. Appl. 76 (1980) 571-599.

[22] A. Rosenfeld, Fuzzy groups, J. Math. Anal. Appl. 35 (1971) 512-517.

[23] M. Shabir, Y.B. Jun, Y. Nawaz, Characterizations of regular semigroups by $(\alpha, \beta)$-fuzzy ideals, Comput. Math. Appl. 59 (2010) 161-175.

[24] M. Shabir, Y.B. Jun, Y. Nawaz, Semigroups characterized by $\left(\in, \in \vee q_{k}\right)$-fuzzy ideals, Comput. Math. Appl. 60 (2010) 1473-1493.

[25] H.S. Vandiver, Note on a simple type of algebra in which cancellation law of addition does not hold, Bull. Amer. Math. Soc. 40 (1934) 914-920.

[26] L. A. Zadeh, Fuzzy sets, Inform. Control 8 (1965) 338-353. 